\title{
Front Matter: Volume 8035
}

, "Front Matter: Volume 8035," Proc. SPIE 8035, Energy Harvesting and Storage: Materials, Devices, and Applications II, 803501 (6 July 2011); doi: 10.1117/12.900055

SPIE Event: SPIE Defense, Security, and Sensing, 2011, Orlando, Florida, United SPIE. States 


\section{PROCEEDINGS OF SPIE}

\section{Energy Harvesting and Storage: Materials, Devices, and Applications II}

Nibir K. Dhar

Priyalal S. Wijewarnasuriya

Achyut K. Dutta

Editors

25-28 April 2011

Orlando, Florida, United States

Sponsored and Published by

SPIE 
The papers included in this volume were part of the technical conference cited on the cover and title page. Papers were selected and subject to review by the editors and conference program committee. Some conference presentations may not be available for publication. The papers published in these proceedings reflect the work and thoughts of the authors and are published herein as submitted. The publisher is not responsible for the validity of the information or for any outcomes resulting from reliance thereon.

Please use the following format to cite material from this book:

Author(s), "Title of Paper," in Energy Harvesting and Storage: Materials, Devices, and Applications II, edited by Nibir K. Dhar, Priyalal S. Wijewarnasuriya, Achyut K. Dutta, Proceedings of SPIE Vol. 8035 (SPIE, Bellingham, WA, 2011) Article CID Number.

ISSN 0277-786X

ISBN 9780819486097

Published by

SPIE

P.O. Box 10, Bellingham, Washington 98227-0010 USA

Telephone +1 3606763290 (Pacific Time) · Fax +1 3606471445

SPIE.org

Copyright (C) 2011, Society of Photo-Optical Instrumentation Engineers

Copying of material in this book for internal or personal use, or for the internal or personal use of specific clients, beyond the fair use provisions granted by the U.S. Copyright Law is authorized by SPIE subject to payment of copying fees. The Transactional Reporting Service base fee for this volume is $\$ 18.00$ per article (or portion thereof), which should be paid directly to the Copyright Clearance Center (CCC), 222 Rosewood Drive, Danvers, MA 01923. Payment may also be made electronically through CCC Online at copyright.com. Other copying for republication, resale, advertising or promotion, or any form of systematic or multiple reproduction of any material in this book is prohibited except with permission in writing from the publisher. The CCC fee code is 0277-786X/11/ \$18.00.

Printed in the United States of America.

Publication of record for individual papers is online in the SPIE Digital Library.

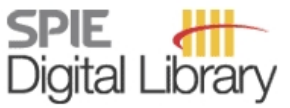

SPIEDigitalLibrary.org

Paper Numbering: Proceedings of SPIE follow an e-First publication model, with papers published first online and then in print and on CD-ROM. Papers are published as they are submitted and meet publication criteria. A unique, consistent, permanent citation identifier (CID) number is assigned to each article at the time of the first publication. Utilization of CIDs allows articles to be fully citable as soon as they are published online, and connects the same identifier to all online, print, and electronic versions of the publication. SPIE uses a six-digit CID article numbering system in which:

- The first four digits correspond to the SPIE volume number.

- The last two digits indicate publication order within the volume using a Base 36 numbering system employing both numerals and letters. These two-number sets start with $00,01,02,03,04$, $05,06,07,08,09,0 A, 0 B \ldots 0 Z$, followed by 10-1Z, 20-2Z, etc.

The CID number appears on each page of the manuscript. The complete citation is used on the first page, and an abbreviated version on subsequent pages. Numbers in the index correspond to the last two digits of the six-digit CID number. 


\section{Contents}

vii Conference Committee

\section{SESSION 1 ADVANCED LITHIUM BATTERIES: ELECTRODES I}

803503 Nanostructured mesoporous materials for lithium-ion battery applications (Invited Paper) [8035-02]

P. Balaya, K. Saravanan, S. Hariharan, V. Ramar, H. S. Lee, M. Kuezma, S. Devaraj,

D. H. Nagaraju, K. Ananthanarayanan, C. W. Mason, National Univ. of Singapore

(Singapore)

803504 Search for greener Li-ion batteries: an alternative offered by organic electroactive materials (Invited Paper) [8035-03]

J. Geng, S. Renault, P. Poizot, F. Dolhem, Univ. de Picardie Jules Verne (France)

\section{SESSION 2 ADVANCED LITHIUM BATTERIES: ELECTRODES II}

803506 Recent advances in nanocrystalline intermetallic tin compounds for the negative electrode of lithium ion batteries (Invited Paper) [8035-05]

R. Alcántara, U. G. Nwokeke, F. Nacimiento, P. Lavela, J. L. Tirado, Univ. de Córdoba (Spain)

803507 Green energy storage materials: advanced nanostructured materials for lithium-ion batteries (Invited Paper) [8035-06]

A. M. Tripathi, M. S. Chandrasekar, S. Mitra, Indian Institute of Technology Bombay (India)

803508 Lithium lanthanum titanate as an electrolyte for novel lithium ion battery systems (Invited Paper) [8035-07]

L. Sun, Univ. of Illinois at Urbana-Champaign (United States) and Massachusetts Institute of Technology (United States); K. Sun, Univ. of Illinois at Urbana-Champaign (United States);

S. Dillon, Massachusetts Institute of Technology (United States)

\section{SESSION 3 ADVANCED LITHIUM BATTERIES: ELECTROLITES}

8035 OB Soft matter electrolytes for Li-ion batteries (Invited Paper) [8035-1 1]

A. J. Bhattacharyya, M. Patel, Indian Institute of Science (India)

8035 OC All-solid-state rechargeable batteries with three-dimensionally ordered macroporous ceramic electrolyte (Invited Paper) [8035-12]

M. Kotobuki, R. Osone, H. Munakata, K. Kanamura, Tokyo Metropolitan Univ. (Japan)

8035 OD An external sensor for instantaneous measurement of the internal temperature in lithium-ion rechargeable cells [8035-13]

R. Srinivasan, B. G. Carkhuff, M. H. Butler, A. C. Baisden, O. M. Uy, The Johns Hopkins Univ. (United States) 
8035 OE All-solid-state thin film microbatteries fabricated by rf sputtering (Invited Paper) [8035-14] J. K. Feng, M. O. Lai, National Univ. of Singapore (Singapore); X. Zeng, Z. Huang, Singapore Institute of Manufacturing Technology (Singapore); L. Lu, National Univ. of Singapore (Singapore)

\section{SESSION 4 ADVANCED CAPACITORS TECHNOLOGY}

8035 OF Pseudo-capacitive reactions based on imidazolium ion (Invited Paper) [8035-15] M. Egashira, T. Tanaka, Y. Matsuno, N. Yoshimoto, M. Morita, Yamaguchi Univ. (Japan)

8035 0G Advances in solid polymer electrochemical capacitors for high rate applications (Invited Paper) [8035-16]

K. Lian, H. Gao, Univ. of Toronto (Canada)

8035 Ol In-situ preparation of PEDOT/ $\mathrm{V}_{2} \mathrm{O}_{5}$ nanocomposite and its synergism for enhanced capacitive behavior [8035-18]

P. Ragupathy, H. N. Vasan, N. Munichandraiah, N. Y. Vasanthacharya, Indian Institute of Science (India)

8035 0J Design, fabrication, and evaluation of on-chip micro-supercapacitors (Invited Paper) [8035-19]

M. Beidaghi, W. Chen, C. Wang, Florida International Univ. (United States)

\section{SESSION 5 PHOTOVOLTAIC CELLS AND RELATED TECHNOLOGIES}

8035 OL Design and simulation of infrared energy harvesting devices [8035-21]

V. Parameshwaran, R. Olah, A. K. Dutta, Banpil Photonics, Inc. (United States); N. K. Dhar, Defense Advanced Research Projects Agency (United States)

8035 OM Nanoscale engineering: optimizing electron-hole kinetics of quantum dot solar cells [8035-22]

K. A. Sablon, U.S. Army Research Lab. (United States); V. Mitin, A. Sergeev, Univ. at Buffalo (United States); J. W. Little, U.S. Army Research Lab. (United States); N. Vagidov, Univ. at Buffalo (United States); K. Reinhardt, Air Force Office for Scientific Research (United States); K. A. Olver, U.S. Army Research Lab. (United States)

8035 ON Metal-black scattering centers to enhance light harvesting by thin-film solar cells [8035-25] D. Panjwani, R. E. Peale, Univ. of Central Florida (United States); I. Oladeji, SISOM Thin Films, LLC (United States); F. K. Rezaie, K. Baillie, J. Colwell, Univ. of Central Florida (United States); C. J. Fredricksen, LRC Engineering Inc. (United States)

\section{SESSION 6 ADVANCED FUEL CELLS}

8035 OR Development of reversible solid oxide fuel cell for power generation and hydrogen production (Invited Paper) [8035-29]

G. B. Jung, J. Y. Chen, C. Y. Lin, S. H. Chan, Yuan Ze Univ. (Taiwan)

8035 OS Recent development of miniatured enzymatic biofuel cell [8035-31]

Y. Song, V. Penmatsa, C. Wang, Florida International Univ. (United States) 
8035 OT Multimodal energy harvesting system (Invited Paper) [8035-32]

V. Bedekar, Virginia Polytechnic Institute and State Univ. (United States); M. Bichurin, I. Solovjev, Novgorod State Univ. (Russian Federation); S. Priya, Virginia Polytechnic Institute and State Univ. (United States)

8035 OU Perpetual harvesting device electronics [8035-33]

R. Olah, G. Mizuno, A. K. Dutta, Banpil Photonics, Inc. (United States); N. K. Dhar, Defense Advanced Research Projects Agency (United States)

8035 OV Development of MEMS based pyroelectric thermal energy harvesters [8035-34] S. R. Hunter, N. V. Lavrik, T. Bannuru, Oak Ridge National Lab. (United States); S. Mostafa, Univ. of Tennessee, Knoxville (United States); S. Rajic, P. G. Datskos, Oak Ridge National Lab. (United States)

8035 OW Innovative microbial fuel cell for energy harvesting and corrosion protection [8035-35] C.-C. Kung, C.-C. Liu, X. Yu, Case Western Reserve Univ. (United States)

8035 0X Ultra-high transmittance through nanostructure-coated glass for solar cell applications [8035-36]

R. E. Welser, A. W. Sood, A. K. Sood, Magnolia Optical Technologies, Inc. (United States) and Magnolia Solar, Inc. (United States); D. J. Poxson, S. Chhajed, J. Cho, E. F. Schubert, Rensselaer Polytechnic Institute (United States); D. L. Polla, N. K. Dhar, Defense Advanced Research Projects Agency (United States)

\section{SESSION 8 ADVANCED THERMO-ELECTRIC DEVICES}

803510 Progress in $\mathrm{Bi}_{2} \mathrm{Te}_{3}$-based superlattice thermoelectric materials (Invited Paper) [8035-39] G. E. Bulman, C. D. Stokes, P. T. Barletta, R. Venkatasubramanian, RTI International (United States)

803511 Method for steady-state, isothermal measurement of thermoelectric materials [8035-40] J. R. Maddux, P. J. Taylor, U.S. Army Research Lab. (United States)

803512 Thin-film thermoelectric energy harvesting for security and sensing applications (Invited Paper) [8035-41]

D. A. Koester, P. Crocco, R. Mahadevan, E. Siivola, K. Von Gunten, Nextreme Thermal Solutions, Inc. (United States)

803514 Solution processible nanoparticle thermoelectric materials [8035-43]

N. Nguyen, G. M. Williams, Jr., T. Allen, D. Schut, Voxtel, Inc. (United States); D. C. Johnson, Univ. of Oregon (United States) 
803516 Energy harvesting from mortar tube firing impulse to supplement fire-control electronics battery [8035-45]

J. Rastegar, R. Murray, Omnitek Partners, LLC (United States); R. Tillinghast, C. Pereira, H.-L. Nguyen, U.S. Army Armament Research, Development and Engineering Ctr. (United States)

803517 Energy-harvesting power sources for gun-fired munitions [8035-46]

J. Rastegar, R. Murray, Omnitek Partners, LLC (United States); C. Pereira, H.-L. Nguyen, U.S. Army Armament Research, Development and Engineering Ctr. (United States)

803519 Energy harvesting roads via pyroelectric effect: a possible approach [8035-49]

A. K. Batra, S. Bhatacharjee, A. K. Chilvery, Alabama A\&M Univ. (United States)

8035 1B Nanocrystal sensitized photovoltaics and photodetectors with performance enhanced using ligand engineering [8035-51]

D. M. Schut, G. M. Williams, Jr., S. Arteaga, T. L. Allen, T. Novet, Voxtel, Inc. (United States)

$80351 \mathrm{C}$ Quantum well and quantum dot energy harvesting devices [8035-52]

R. E. Welser, A. K. Sood, Magnolia Optical Technologies, Inc. (United States) and Magnolia Solar, Inc. (United States); O. A. Laboutin, Kopin Corp. (United States); L. J. Guido, Virginia Polytechnic Institute and State Univ. (United States); N. K. Dhar, Defense Advanced Research Projects Agency (United States); P. S. Wijewarnasuriya, U.S. Army Research Lab. (United States)

8035 1D A high-temperature acoustic-electric system for power delivery and data communication through thick metallic barriers [8035-53]

T. J. Lawry, K. R. Wilt, S. Roa-Prada, J. D. Ashdown, G. J. Saulnier, H. A. Scarton, Rensselaer Polytechnic Institute (United States); P. K. Das, Univ. of California, San Diego (United States); A. J. Gavens, Bechtel Marine Propulsion Corp. (United States)

Author Index 


\title{
Conference Committee
}

\author{
Symposium Chair
}

William Jeffrey, HRL Laboratories, LLC (United States)

Symposium Cochair

Kevin P. Meiners, Office of the Secretary of Defense (United States)

Conference Chairs

Nibir K. Dhar, Defense Advanced Research Projects Agency (United States)

Priyalal S. Wijewarnasuriya, U.S. Army Research Laboratory (United States)

Achyut K. Dutta, Banpil Photonics, Inc. (United States)

\section{Program Committee}

Pulickel M. Ajayan, Rice University (United States)

Palani Balaya, National University of Singapore (Singapore)

Fow-Sen Choa, University of Maryland, Baltimore County (United States)

Deryn Chu, U.S. Army Research Laboratory (United States)

Angelo S. Gilmore, EPIR Technologies, Inc. (United States)

M. Saif Islam, University of California, Davis (United States)

Ahalapitiya H. Jayatissa, The University of Toledo (United States)

Nobuhiko P. Kobayashi, University of California, Santa Cruz (United States)

Pat McGrath, Booz Allen Hamilton Inc. (United States)

Robert Olah, Banpil Photonics, Inc. (United States)

Unil A. Perera, Georgia State University (United States)

A. Fred Semendy, U.S. Army Research Laboratory (United States)

Ashok K. Sood, Magnolia Optical Technologies, Inc. (United States)

Rao Surampudi, Jet Propulsion Laboratory (United States)

Patrick J. Taylor, U.S. Army Research Laboratory (United States)

Sudhir B. Trivedi, Brimrose Corporation of America (United States)

Rama Venkatasubramanian, RTI International (United States)

Chunlei Wang, Florida International University (United States) 
Session Chairs

1 Advanced Lithium Batteries: Electrodes I

Nibir K. Dhar, Defense Advanced Research Projects Agency (United States)

Achyut Dutta, Banpil Photonics, Inc. (United States)

2 Advanced Lithium Batteries: Electrodes II

Palani Balaya, National University of Singapore (Singapore)

3 Advanced Lithium Batteries: Electrolites

Palani Balaya, National University of Singapore (Singapore)

$4 \quad$ Advanced Capacitors Technology

Palani Balaya, National University of Singapore (Singapore)

Minato Egashira, Yamaguchi University (Japan)

$5 \quad$ Photovoltaic Cells and Related Technologies

Nibir K. Dhar, Defense Advanced Research Projects Agency (United States)

Achyut Dutta, Banpil Photonics, Inc. (United States)

6 Advanced Fuel Cells

Palani Balaya, National University of Singapore (Singapore)

$7 \quad$ Advanced Harvesting Devices

Priyalal S. Wijewarnasuriya, U.S. Army Research Laboratory (United States)

Achyut Dutta, Banpil Photonics, Inc. (United States)

8 Advanced Thermo-Electric Devices

Gary E. Bulman, RTI International (United States)

Patrick J. Taylor, U.S. Army Research Laboratory (United States)

9 Nanotechnologies for Energy Generation and Storage I: Joint Session with Conference 8031

Jeremy J. Pietron, U.S. Naval Research Laboratory (United States)

Nezih Pala, Florida International University (United States)

10 Nanotechnologies for Energy Generation and Storage II: Joint Session with Conference 8031

Jeremy J. Pietron, U.S. Naval Research Laboratory (United States)

Nezih Pala, Florida International University (United States) 